\title{
Impact of Magnetic Fields on Fragmentation
}

\author{
Alan P. Boss \\ Carnegie Institution of Washington, Department of Terrestrial \\ Magnetism, 5241 Broad Branch Road, N. W., Washington, D. C. \\ 20015-1305, U.S.A.
}

\begin{abstract}
Fragmentation is the leading explanation for the formation of binary and multiple stars. However, nearly all three dimensional calculations of the collapse and fragmentation of dense molecular cloud cores have ignored the effects of magnetic fields, whereas magnetic fields are generally regarded to be a dominant force in molecular clouds. Three dimensional models of the collapse of clouds with frozen-in magnetic fields have shown that such clouds cannot fragment for a range of initial conditions. However, calculations that allow for magnetic field loss by ambipolar diffusion have shown that fragmentation is possible for initially prolate or oblate, rotating, magnetically-supported cloud cores. The latter calculations rely on approximations that should be verified by more detailed, traditional magnetohydrodynamical codes. The most obvious effect of magnetic fields is to delay the onset of the collapse phase, but once collapse begins in earnest, fragmentation proceeds in much the same manner as in nonmagnetic clouds, with initially prolate clouds tending to form binary protostars, and with initially oblate clouds tending to form multiple protostars.
\end{abstract}

\section{Introduction}

Several mechanisms have been proposed to account for binary star formation, but fragmentation has been recognized as the leading mechanism for most of the last decade (see reviews by Boss 1988, 1993; Pringle 1991; Bodenheimer et al. 1993; Bodenheimer 1995; Bodenheimer et al. 2000). Recent observational work appears to have largely confirmed the assertion that fragmentation is the dominant mechanism for binary star formation, and studies presented at this symposium have only strengthened this conclusion. The discovery that the binary frequency among pre-main-sequence stars (Ghez et al. 1993; Leinert et al. 1993; Reipurth \& Zinnecker 1993; Richichi et al. 1994; Simon et al. 1995; Brandner et al. 1996; Petr et al. 1998; Duchêne et al. 1999) is comparable to or greater than that among nearby main-sequence stars (Duquennoy \& Mayor 1991; Fischer \& Marcy 1992) requires that most binary stars be formed prior to the pre-mainsequence phase, i.e., during the protostellar collapse phase. This observational fact points directly at fragmentation as the most likely formation mechanism. At the same time, detailed observations of pre-collapse molecular cloud cores show that they have properties conducive to the occurrence of fragmentation 
during collapse (Myers et al. 1991; Goodman et al. 1993; Ward-Thompson et al. 1994; André et al. 1996; Ward-Thompson et al. 1999).

In spite of this rosy observational picture, relatively little theoretical effort has been expended in understanding the role of magnetic fields in the threedimensional collapse and fragmentation process. A fair amount of theoretical effort has gone into calculating the effects of magnetic fields on the contraction and early collapse phases of axisymmetric (two dimensional or 2D) clouds, which flatten into pancakes as a result of the effects of an initially aligned, uniform magnetic field (e.g., Mouschovias 1987, 1991; Nakano \& Umebayashi 1988; Tomisaka et al. 1988, 1990; Lizano \& Shu 1989; Ciolek \& Mouschovias 1995). The 2D studies serve as invaluable guides to what could be expected in a fully three-dimensional (3D) calculation, but in the absence of 3D studies, it is hard to predict whether magnetic effects will help or hinder the fragmentation mechanism. The purpose of this review is to summarize recent theoretical work that attempts to include the effects of magnetic fields in at least a provisional manner in $3 \mathrm{D}$ collapse calculations, in order to assess the impact of magnetic fields on fragmentation.

\section{Observations of Pre-Collapse Clouds}

The standard theory of star formation (Shu et al. 1987) postulates that dense cloud cores are magnetically supported, and contract because of ambipolar diffusion toward the singular isothermal sphere, which then undergoes inside-out collapse (Shu 1977) leading to the formation of a single protostar. This theory has been remarkably successful in explaining many properties of young stars, but the need for significant modification has arisen, not only from the point of view of the question of the origin of binary stars, but also from studies of the interstellar medium.

Recently Tafalla et al. (1998) and Williams et al. (1999) found evidence for large-scale inward motion in the starless L1544 cloud, with velocities of 0.1 $\mathrm{km} / \mathrm{sec}$ extending over a region of size $0.1 \mathrm{pc}$. They pointed out that such largescale infall in a pre-collapse cloud was formally inconsistent with the standard theory of ambipolar diffusion followed by inside-out collapse. Similarly, Williams \& Myers (1999) found inward motions of $0.3 \mathrm{~km} / \mathrm{sec}$ over an extended region of a Serpens starless cloud. Nakano (1998) has argued that supersonic turbulence and magnetic fields contribute equally to cloud core support, so that cloud cores are magnetically supercritical, rather than subcritical as advanced by Shu et al. (1987). Crutcher (1999) reviewed all of the Zeeman data on magnetic fields in molecular clouds, and concluded that the supersonic motions are likely to be Alfvén waves, with the kinetic energy of the turbulence being roughly equal to the magnetic energy, implying equal support. This turbulence is probably driven largely by protostellar outflows. Once the turbulence decays in a given cloud, contraction and collapse can then begin (Nakano 1998).

The need for another modification to the standard theory arises from the density profiles inferred for pre-collapse clouds. Many dense cloud cores were found to be strongly centrally concentrated, with inferred power-law density profiles similar to those of the singular isothermal sphere $\left(\rho \propto r^{-2}\right)$ or of its inner collapsing region $\left(\rho \propto r^{-3 / 2}\right)$. However, these cloud cores contained embedded 
young stars and so could not be pre-collapse clouds. Ward-Thompson et al. (1994) found that starless cores were less centrally peaked than cores containing embedded objects. In fact, Ward-Thompson et al. (1994) found that a Gaussian density profile provided the best fit for starless cores. Subsequent observations with higher spatial resolution confirm that pre-collapse clouds have flat density profiles at their centers (André et al. 1996; Ward-Thompson et al. 1999), rather than the power-law profiles expected in the standard theory. Initial Gaussian density profiles were first employed in 3D fragmentation calculations by Boss (1987), and were found to enhance greatly the prospects for fragmentation compared to power-law initial density profiles.

Myers et al. (1991) studied the projected shapes of dense cloud cores, and concluded that most cloud cores are likely to be prolate with axis ratios of 2:1, while some are oblate. Goodman et al. (1993) estimated the amount of solidbody rotation in dense cloud cores, and found that while few clouds showed evidence for centrifugal forces strong enough to affect their pre-collapse structure, most clouds contain enough angular momentum to result in the formation of a binary star system or a large protostellar disk. Ohashi et al. (1997) found a break in the specific angular momentum $(\mathrm{J} / \mathrm{M})$ spectrum of cloud envelopes at $0.03 \mathrm{pc}$ radius (about $1 M_{\odot}$ ), implying that smaller radius clouds collapse with roughly conserved J/M. Taken together, these sorts of observations define the initial conditions for protostellar collapse calculations.

\section{Magnetic Cloud Collapse Calculations}

While many workers have studied the contraction of magnetic clouds (e.g., Mouschovias 1987, 1991; Nakano \& Umebayashi 1988; Tomisaka et al. 1988; Lizano \& Shu 1989; Nakamura \& Hanawa 1997), only a handful of papers have attempted to include magnetic fields in fully $3 \mathrm{D}$ protostellar collapse calculations. Most of these have focused on frozen-in magnetic fields (Dorfi 1982; Benz 1984; Phillips \& Monaghan 1985; Phillips 1986a, 1986b), and all agreed that clouds with frozen-in magnetic fields show no tendency to fragment into binary protostars. In particular, Phillips (1986a) examined the collapse of nonrotating clouds with a wide range of initial thermal $\left(\alpha_{i}=E_{\text {therm }} /\left|E_{\text {grav }}\right|=0.06\right.$ to 0.95$)$ and magnetic energies $\left(\gamma_{i}=E_{\text {mag }} /\left|E_{\text {grav }}\right|=0.006\right.$ to 0.6$)$, and found no evidence for fragmentation, even when the cloud started with a quite large density perturbation. Dorfi (1982) found a similar result for a rotating, strongly magnetic cloud. These calculations suggested, therefore, that it was unlikely that fragmentation could explain the formation of binary stars in magnetic molecular cloud cores.

However, frozen-in magnetic fields are more of a theoretical simplification than a realistic description of dense cloud cores. The standard theory of star formation envisions that dense cloud cores contract over time periods on the order of $10 \mathrm{Myr}$ as a result of ambipolar diffusion (Shu et al. 1987), which eventually leads to a dynamic collapse phase similar to that assumed in most nonmagnetic collapse calculations, so it is important to reconsider whether fragmentation could occur in a magnetic 3D cloud undergoing ambipolar diffusion.

Boss $(1997,1999)$ presented 3D collapse calculations using the magnetic pressure approximation (see below) and a parameterized treatment of ambipolar 
diffusion based on detailed models by Ciolek \& Mouschovias (1995). The initial clouds were prolate, with 2:1 axis ratios (Myers et al. 1991), and had a Gaussian density profile with a flat inner region (e.g., André et al. 1996). The clouds were rotating initially with either solid body or differential rotation, with ratios of rotational to gravitational energies $\left(\beta_{i}\right)$ ranging from $\approx 10^{-4}$ to 0.1 , spanning the observed range for cloud cores (Goodman et al. 1993). Basu \& Mouschovias (1994) showed that the central regions of magnetically-contracting clouds are likely to be in differential rotation with angular velocity increasing as $\Omega \propto \rho^{1 / 2}$. With an initial ratio of thermal to gravitational energy of $\alpha_{i}=0.39$, these clouds would have collapsed and fragmented in the absence of magnetic effects. Radiative transfer in the Eddington approximation was included in the models.

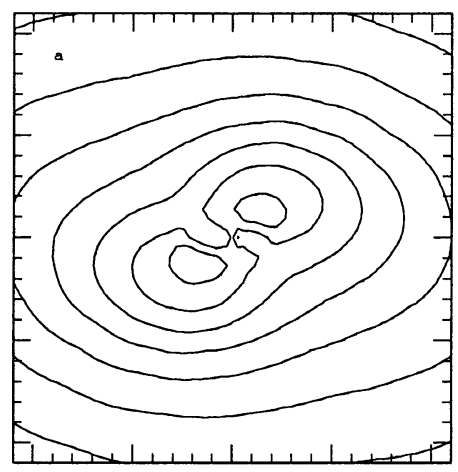

Fig. 1. Equatorial density for a magnetic cloud core with $\beta_{i}=0.012$ at $t=$ $10.344 t_{f f}, \rho_{\max }=1.6 \times 10^{-12} \mathrm{~g} \mathrm{~cm}^{-3}$, box radius $=6.7 \times 10^{14} \mathrm{~cm}$. A binary protostar forms. Contours denote factor of two changes.

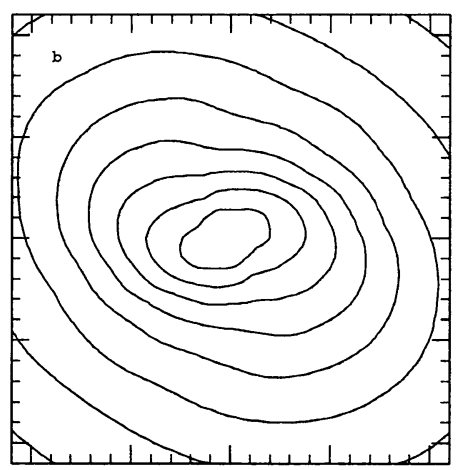

Fig. 2. Equatorial density for a magnetic cloud core with $\beta_{i}=0.008$ at $t=$ $10.255 t_{f f}, \rho_{\max }=1.6 \times 10^{-12} \mathrm{~g} \mathrm{~cm}^{-3}$, box radius $=6.4 \times 10^{14} \mathrm{~cm}$. A bar-like protostar forms, but binary fragmentation does not occur (cf. Fig. 1). 
Figures 1 and 2 demonstrate the most important outcome of these magnetic cloud models - the dependence on the initial rotation rate. While varying the timescale for ambipolar diffusion or the degree of differential rotation had little effect, the results were quite dependent on $\beta_{i}$ : clouds starting with $\beta_{i}>0.01$ fragmented into binary protostars, while clouds with $\beta_{i}<0.01$ seemed to form only bars or single protostars (Boss 1999). These models showed that fragmentation can still occur in magnetic clouds that evolve through loss of magnetic field support by ambipolar diffusion, provided that the initial cloud rotation rate is high enough. The main effect of the magnetic field was to delay the dynamic collapse phase, and hence the fragmentation phase, until ambipolar diffusion had reduced the magnetic support sufficiently.

\section{Magnetic Field Approximations}

The problem of fragmentation in a magnetic cloud subject to ambipolar diffusion should be studied with a true magnetohydrodynamics (MHD) code, such as the ZEUS code (Stone \& Norman 1992; see also Hujeirat et al. 2000 and Indebetouw \& Zweibel 2000). In the meantime, considerable insight can be gained by including the effects of magnetic fields through several reasonably good approximations (e.g., Boss 1997, 1999). The validity of these approximations can be assessed as follows. We start with the full MHD momentum equation,

$$
\rho \frac{\partial \vec{v}}{\partial t}+\rho \vec{v} \cdot \nabla \vec{v}=-\rho \nabla \Phi-\nabla p+\frac{1}{c}(\vec{J} \times \vec{B}),
$$

where $\rho=$ density, $\vec{v}=$ velocity, $\Phi=$ gravitational potential, $p=$ gas pressure, $c=$ speed of light, $\vec{J}=$ current density, and $\vec{B}=$ magnetic field.

In the limit of high electrical conductivity, as is the case for molecular cloud cores in spite of their low fractional ionization, Ampére's law holds, so that

$$
\frac{1}{c} \vec{J}=\frac{1}{4 \pi}(\nabla \times \vec{B}) \text {. }
$$

The magnetic effects term then becomes

$$
\frac{1}{c}(\vec{J} \times \vec{B})=-\nabla\left(\frac{B^{2}}{8 \pi}\right)+\frac{1}{4 \pi}(\vec{B} \cdot \nabla) \vec{B} .
$$

For a straight, single component magnetic field $\vec{B}=\left(0,0, B_{z}(x, y)\right)$, the magnetic field tension term $(\vec{B} \cdot \nabla) \vec{B}$ vanishes, leaving

$$
\frac{1}{c}(\vec{J} \times \vec{B})=-\nabla\left(\frac{B^{2}}{8 \pi}\right) .
$$

The full MHD momentum equation then becomes

$$
\rho \frac{\partial \vec{v}}{\partial t}+\rho \vec{v} \cdot \nabla \vec{v}=-\rho \nabla \Phi-\nabla\left(p+\frac{B^{2}}{8 \pi}\right) .
$$

We can thus approximate the effects of magnetic fields simply by adding the effective magnetic pressure term $\left(B^{2} / 8 \pi\right)$ in to the gas pressure. In fact, the 
magnetic pressure formalism is exact for high conductivities and straight field lines, not just an approximation. Calculations of the $2 \mathrm{D}$ contraction of magnetic clouds constrained to axisymmetry show that initially straight magnetic field lines remain remarkably straight throughout the ambipolar diffusion phase, and only begin bending once dynamic collapse begins near the center of the cloud (e.g., Fiedler \& Mouschovias 1993), suggesting that this approximation might be quite accurate, at least for the early phases of contraction. Observations of star-forming regions also support the existence of straight magnetic field lines on relatively large scales.

In the models calculated by Boss $(1997,1999)$, the local magnetic field strength is assumed to depend on the density $\rho$ as

$$
B(\rho, t)=B_{o}(t)\left[\rho / \rho_{o}\right]^{\kappa},
$$

where $B_{o}$ and $\rho_{o}$ are the initial central values and $\kappa=1 / 2$, as is found in detailed MHD calculations (e.g., Tomisaka et al. 1990; Ciolek \& Mouschovias 1995). Crutcher (1999) found that field strengths obtained from Zeeman observations of molecular clouds varied with density as $\rho^{\kappa}$, with $\kappa \approx 0.47$, so this approximation appears to be well-founded as well.

Ambipolar diffusion in Boss $(1997,1999)$ was treated by assuming that

$$
B_{o}(t)=B_{o i}\left[1-t / t_{A D}\right],
$$

where $B_{o i}$ is a constant, $t$ is the model time, and the timescale for ambipolar diffusion $t_{A D}$ is either 10 or $20 t_{f f}$, consistent with previous estimates (e.g., Lizano \& Shu 1989; Tomisaka et al. 1990; Mouschovias 1991). This simple approximation led to a dependence of the central density on time that is quite similar to that found in the detailed 2D models of Ciolek \& Mouschovias (1995).

However, once the collapse phase is well underway, magnetic field lines can bend significantly and exert a tension force that counteracts gravity. Magnetically-controlled clouds tend to contract and flatten along the magnetic field lines and form thin disks. For a thin disk with a constant mass-to-flux ratio $\mu$, the magnetic acceleration $a_{m}$ can be expressed as (Basu 1997, 1998; Shu \& Li 1997; Nakamura \& Hanawa 1997)

$$
a_{m}=a_{\text {tension }}+a_{\text {pressure }}=-\frac{1}{\mu^{2}} g_{r}+\frac{2}{\mu^{2}} a_{T},
$$

where $g_{r}$ is the gravitational acceleration and $a_{T}$ is the thermal acceleration. The magnetic tension acceleration $a_{t e n s i o n}$ thus can be written as

$$
a_{\text {tension }}=-\frac{1}{2} \frac{g_{r}}{a_{T}} a_{\text {pressure }}=-\frac{1}{2} \nabla \Phi \frac{\nabla \frac{B^{2}}{8 \pi}}{\nabla p} .
$$

Hence we can include the magnetic tension acceleration simply by modifying the gravitational potential $\Phi$ as follows

$$
\nabla \Phi \rightarrow\left(1-\frac{1}{2} \frac{\nabla \frac{B^{2}}{8 \pi}}{\nabla p}\right) \nabla \Phi
$$


For an isothermal gas and a magnetic field that varies with density as $B \propto \rho^{\kappa}$, with $\kappa=1 / 2$, the gradient of the density occurs in both the numerator and denominator and cancels out, leaving

$$
\frac{\nabla \frac{B^{2}}{8 \pi}}{\nabla p}=\frac{p_{m a g}(t)}{p(t=0)}=f(t)
$$

Hence once a thin magnetic disk forms, magnetic tension forces can be approximated by diluting the gravitational potential by a factor involving a function only of time

$$
\Phi \rightarrow \Phi \times\left(1-\frac{1}{2} f(t)\right)
$$

where $f(t)$ decreases with time due to the effects of ambipolar diffusion. The field tension approximation can be used in 3D models to provide a more accurate approximation of magnetic effects than is obtained by including only the magnetic pressure. Because magnetic tension dilutes the gravitational attraction which drives fragmentation in the first place, investigating the effects of the magnetic tension approximation could be quite important for the fragmentation mechanism.

\section{3D Magnetic Tension Models}

Nakamura \& Hanawa (1997) have used the thin disk approximation to study the growth of asymmetry in an infinitely thin, rotating, magnetically-supported disk. They found that such a disk can become quite bar-like, provided that an initial asymmetry is present, and that it is therefore likely to fragment into two or more protostars.

Figure 3 shows the result of a 3D model of magnetic cloud collapse identical to that of Figure 2, except that the field tension approximation is employed as well as the magnetic pressure approximation (and $\tau_{a d}$ is $10 t_{f f}$ instead of $20 t_{f f}$ ). Comparing Figures 3 and 2, it is evident that adding field tension effects has led to fragmentation in a cloud that otherwise did not fragment because of its low initial rotation rate. Figure 4 shows that an initially oblate cloud with a very low rotation rate stills fragments, in this case into a quadruple system, when field tension is included. Apparently field tension effects encourage rather than discourage fragmentation in these models, all of which obey the Jeans conditions (Truelove et al. 1997) for a spherical coordinate grid (Boss 1998).

These models suggest that the typical outcome of the collapse of a magnetic cloud is either a binary or multiple protostar. If verified, these results would then imply that the formation of a single protostar is a relatively rare event, and that most single stars must result from the decay of multiple protostar systems. 


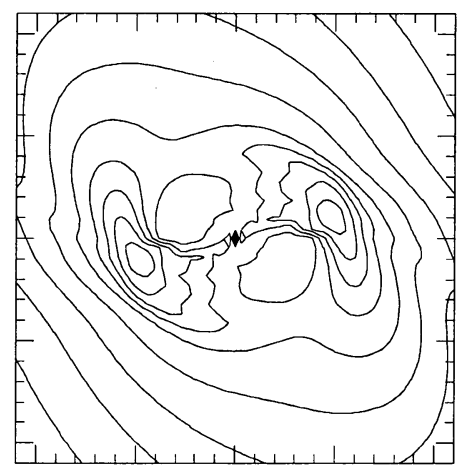

Fig. 3. Equatorial density for an initially prolate, magnetic cloud core with $\beta_{i}=0.008$ at $t=5.146 t_{f f}, \rho_{\max }=4.0 \times 10^{-12} \mathrm{~g} \mathrm{~cm}^{-3}$, box radius $=8.2 \times 10^{14}$ $\mathrm{cm}$. Magnetic tension effects are included. A binary protostar forms.

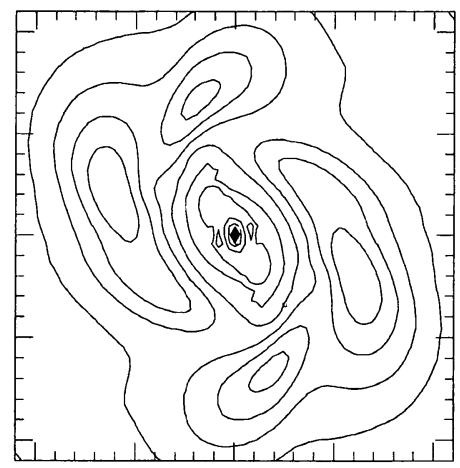

Fig. 4. Equatorial density for an initially oblate, magnetic cloud core with $\beta_{i}=0.0001$ at $t=2.615 t_{f f}, \rho_{\max }=7.9 \times 10^{-10} \mathrm{~g} \mathrm{~cm}^{-3}$, box radius $=3.5 \times 10^{13}$ $\mathrm{cm}$. Magnetic tension effects are included. A quadruple protostar forms.

\section{Conclusions}

Theoretical models of the 3D collapse of molecular clouds with magnetic field support indicate that fragmentation is prohibited when the magnetic fields are frozen-in, but is permissible when ambipolar diffusion is allowed to reduce the magnetic contribution to the cloud's support prior to the onset of the collapse phase. New 3D models employing the magnetic pressure and field tension approximations described here imply that dense clouds with a wide range of initial rotation rates will fragment, contrary to the situation where field tension effects are ignored, where only rapidly-rotating clouds seem to fragment. These models thus suggest that magnetic fields can encourage fragmentation. Initially prolate 
clouds tend to fragment into binary protostars, while initially oblate clouds tend to form multiple protostar systems, at least at this very early phase.

The subsequent evolution and possible survival of these binary and multiple protostellar systems remain to be determined and is a significant challenge for future work. In order to achieve the highest possible fidelity to the physical conditions in dense molecular clouds, as determined by observations, future calculations should include the combined effects of magnetic fields and turbulent velocity fields. Inclusion of turbulent motions also seems to aid in the fragmentation process (Klein et al., this volume), lending credence to the hope that in the ultimate physical description of the collapse of a dense molecular cloud core, fragmentation will remain as the leading mechanism for explaining the formation of the great majority of binary and multiple star systems.

\section{References}

André, P., Ward-Thompson, D., Motte, F. 1996, A\&A, 314, 625

Basu, S. 1997, ApJ, 485, 240

Basu, S. 1998, ApJ, 509, 229

Basu, S., Mouschovias, T. Ch. 1994, ApJ, 432, 720

Benz, W. 1984, A\&A, 139, 378

Bodenheimer, P. 1995, ARA\&A, 33, 199

Bodenheimer, P., Burkert, A., Klein, R. I., Boss, A. P. 2000, in Protostars and Planets IV, ed. V. G. Mannings, A. P. Boss, S. S. Russell (University of Arizona Press: Tucson), 675

Bodenheimer, P., Ruzmaikina, T., Mathieu, R. D. 1993, in Protostars \& Planets III, ed. E. H. Levy, J. I. Lunine (University of Arizona Press: Tucson), 367

Boss, A. P. 1987, ApJ, 319, 149

Boss, A. P. 1988, Comm. Astrophys., 12, 169

Boss, A. P. 1993, in The Realm of Interacting Binary Stars, ed. J. Sahade, G. McCluskey, Y. Kondo (Kluwer: Dordrecht), 355

Boss, A. P. 1997, ApJ, 483, 309

Boss, A. P. 1998, ApJ, 501, L77

Boss, A. P. 1999, ApJ, 520, 744

Brandner, W., Alcalá, J. M., Kunkel, M., Moneti, A., Zinnecker, H. 1996, A\&A, 307,121

Ciolek, G. E., Mouschovias, T. Ch. 1995, ApJ, 454, 194

Crutcher, R. M. 1999, ApJ, 520, 706

Dorfi, E. 1982, A\&A, 114, 151

Duchêne, G., Bouvier, J., Simon, T. 1999, A\&A, 343, 831

Duquennoy, A., Mayor, M. 1991, A\&A, 248, 485

Fiedler, R. A., Mouschovias, T. Ch. 1993, ApJ, 415, 680

Fischer, D. A., Marcy, G. W. 1992, ApJ, 396, 178

Ghez, A. M., Neugebauer, G., Matthews, K. 1993, AJ, 106, 2005 
Goodman, A. A., Benson, P. J., Fuller, G. A., Myers, P. C. 1993, ApJ, 406, 528 Hujeirat, A., Camenzind, M., Yorke, H. W. 2000, A\&A, 354, 1041

Indebetouw, R., Zweibel, E. G. 2000, ApJ, 532, 361

Leinert, C., Zinnecker, H., Weitzel, N., Christou, J., Ridgway, S. T., Jameson, R., Haas, M., Lenzen, R. 1993, A\&A, 278, 129

Lizano, S., Shu, F. H. 1989, ApJ, 342, 834

Mouschovias, T. Ch. 1987, in Physical Processes in Interstellar Clouds, ed. G. E. Morfill, M. Scholer (D. Reidel: Dordrecht), 453

Mouschovias, T. Ch. 1991, ApJ, 373, 169

Myers, P. C., Fuller, G. A., Goodman, A. A., Benson, P. J. 1991, ApJ, 376, 561 Nakamura, F., Hanawa, T. 1997, ApJ, 480, 701

Nakano, T. 1998, ApJ, 494, 587

Nakano, T., Umebayashi, T. 1988, Progr. Theor. Phys. Suppl., 96, 73

Ohashi, N., Hayashi, M., Ho, P. T. P., Momose, M., Tamura, M., Hirano, N., Sargent, A. I. 1997, ApJ, 488, 317

Petr, M. G., Du Foresto, V. C., Beckwith, S. V. W., Richichi, A., McCaughrean, M. J. 1998, ApJ, 500, 825

Phillips, G. J. 1986a, MNRAS, 221, 571

Phillips, G. J. 1986b, MNRAS, 222, 111

Phillips, G. J., Monaghan, J. J. 1985, MNRAS, 216, 883

Pringle, J. E. 1991, in The Physics of Star Formation and Early Stellar Evolution, ed. C. J. Lada, N. D. Kylafis (Kluwer: Dordrecht), 437

Reipurth, B., Zinnecker, H. 1993, A\&A, 278, 81

Richichi, A., Leinert, C., Jameson, R., Zinnecker, H. 1994, A\&A, 287, 145

Shu, F. H. 1977, ApJ, 214, 488

Shu, F. H., Adams, F. C., Lizano, S. 1987, ARA\&A, 25, 23

Shu, F. H., Li, Z.-Y. 1997, ApJ, 475, 251

Simon, M., Ghez, A. M., Leinert, C. H., Cassar, L., Chen, W. P., Howell, R. R., Jameson, R. F., Matthews, K. 1995, ApJ, 443, 625

Stone, J. M., Norman, M. L. 1992, ApJS, 80, 791

Tafalla, M., Mardones, D., Myers, P. C., Caselli, P., Bachiller, R., Benson, P. J. 1998, ApJ, 504, 900

Tomisaka, K., Ikeuchi, S., Nakamura, T. 1988, ApJ, 326, 208

Tomisaka, K., Ikeuchi, S., Nakamura, T. 1990, ApJ, 362, 202

Truelove, J. K., Klein, R. I., McKee, C. F., Holliman, J. H., Howell, L. H., Greenough, J. A. 1997, ApJ, 489, L179

Ward-Thompson, D., Motte, F., André, P. 1999, MNRAS, 305, 143

Ward-Thompson, D., Scott, P. F., Hills, R. E., André, P. 1994, MNRAS, 268, 276

Williams, J. P., Myers, P. C. 1999, ApJ, 518, L37

Williams, J. P., Myers, P. C., Wilner, D. J., Di Francesco, J. 1999, ApJ, 513, L61 